\title{
Photospheric activity, rotation, and radial velocity variations of the planet-hosting star CoRoT-7 ${ }^{\star}$
}

\author{
A. F. Lanza ${ }^{1}$, A. S. Bonomo ${ }^{1,2}$, C. Moutou ${ }^{2}$, I. Pagano ${ }^{1}$, S. Messina ${ }^{1}$, G. Leto ${ }^{1}$, G. Cutispoto ${ }^{1}$, S. Aigrain ${ }^{3}$, R. Alonso ${ }^{4}$,

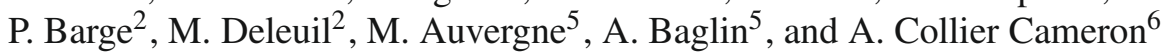

1 INAF - Osservatorio Astrofisico di Catania, via S. Sofia 78, 95123 Catania, Italy e-mail: nuccio.lanza@oact.inaf.it

${ }^{2}$ Laboratoire d'Astrophysique de Marseille (UMR 6110), Technopole de Château-Gombert, 38 rue Frédéric Joliot-Curie, 13388 Marseille Cedex 13, France

3 Department of Physics, University of Oxford, Denys Wilkinson Building, Keble Road, Oxford OX1 3RH, UK

4 Observatoire de Genève, Université de Genève, 51 Ch. des Maillettes, 1290 Sauverny, Switzerland

5 LESIA, CNRS UMR 8109, Observatoire de Paris, 5 place J. Janssen, 92195 Meudon, France

${ }^{6}$ School of Physics and Astronomy, University of St. Andrews, North Haugh, St Andrews, Fife KY16 9SS, Scotland

Received 11 March 2010 / Accepted 17 May 2010

\section{ABSTRACT}

\begin{abstract}
Context. The CoRoT satellite has recently discovered the transits of an Earth-like planet across the disc of a late-type magnetically active star dubbed CoRoT-7, while a second planet was detected after filtering out the radial velocity (hereafter RV) variations due to stellar activity.

Aims. We investigate the magnetic activity of CoRoT-7 and use the results for a better understanding of the impact of magnetic activity on stellar RV variations.

Methods. We derived the longitudinal distribution of active regions on CoRoT-7 from a maximum entropy spot model of the CoRoT lightcurve. Assuming that each active region consists of dark spots and bright faculae in a fixed proportion, we synthesized the expected RV variations.

Results. Active regions are mainly located at three active longitudes that appear to migrate at different rates, probably as a consequence of surface differential rotation, for which a lower limit of $\Delta \Omega / \Omega=0.058 \pm 0.017$ is found. The synthesized activity-induced RV variations reproduce the amplitude of the observed RV curve and are used to study the impact of stellar activity on planetary detection.

Conclusions. In spite of the non-simultaneous CoRoT and HARPS observations, our study confirms the validity of the method previously adopted to filter out RV variations induced by stellar activity. We find a false-alarm probability $<10^{-4}$ that the RV oscillations attributed to CoRoT-7b and CoRoT-7c are spurious effects of noise and activity. Additionally, our model suggests that other periodicities found in the observed RV curve of CoRoT-7 could be explained by active regions whose visibility is modulated by a differential stellar rotation with periods ranging from 23.6 to 27.6 days.
\end{abstract}

Key words. stars: activity - stars: magnetic field - stars: late-type - stars: rotation - planetary systems - stars: individual: CoRoT-7

\section{Introduction}

CoRoT (Convection, Rotation and Transits) is a photometric space experiment devoted to asteroseismology and the search for extrasolar planets by the method of transits (Baglin et al. 2006). It has recently discovered two Earth-like planets around a latetype star called CoRoT-7, the innermost of which transits across the disc of the star and has an orbital period of only 0.8536 days, while the other has a period of 3.69 days and does not show transits (Léger et al. 2009; Queloz et al. 2009). The mass of the inner planet CoRoT-7b is $4.8 \pm 0.8 M_{\oplus}$, while that of CoRoT-7c is $8.4 \pm 0.9 M_{\oplus}$, assuming they are on coplanar orbits (Queloz et al. 2009). The relative depth of the transit of CoRoT-7b is $\Delta F / F=(3.35 \pm 0.12) \times 10^{-4}$ in the CoRoT white passband (see Sect. 2), leading to a planetary radius of $1.68 \pm 0.09 R_{\oplus}$.

* Based on observations obtained with CoRoT, a space project operated by the French Space Agency, CNES, with partecipation of the Science Programme of ESA, ESTEC/RSSD, Austria, Belgium, Brazil, Germany, and Spain.
CoRoT-7 is an active star showing a rotational modulation of its optical flux with an amplitude up to $\sim 0.03$ mag and a period of 23.5 days (Queloz et al. 2009). The discovery of CoRoT-7c and the measurement of the mass of CoRoT-7b have become possible with the HARPS spectrograph after filtering out the apparent variations of the stellar RV induced by magnetic activity which have an amplitude of $\approx 40 \mathrm{~m} \mathrm{~s}^{-1}$, i.e., 5-6 times greater than the wobbles produced by the gravitational pull of the planets. Therefore, a more detailed model of the stellar activity is needed to improve RV measurements and to obtain better planetary parameters. This would also rule out the possibility that a quasi-periodic RV signal is caused by rotating spots on the stellar surface instead of a planetary companion as, e.g., in the case of HD 166435 (Queloz et al. 2001).

We applied the same spot modelling approach as used for the high-precision lightcurves of CoRoT-2 (Lanza et al. 2009a) and CoRoT-4 (Lanza et al. 2009b) to derive the longitudinal distribution of photospheric brightness inhomogeneities and their evolution during the $\sim 130$ days of CoRoT observations. The 
different rotation rates of active regions allowed us to estimate a lower limit for the amplitude of the differential rotation of CoRoT-7. From our spot maps, we synthesized the activityinduced RV perturbations using a simple model for the distortion of the line profiles, as described in Sect. 3.2. A Fourier analysis of such a synthetic time series yielded typical periods induced by stellar activity, allowing us to discuss its impact on the detection of planets around CoRoT-7.

\section{Observations}

CoRoT-7 was observed during the first long run of CoRoT toward the galactic anticentre from 24 October 2007 to 3 March 2008. Since the star is bright $(V=11.67)$, the time sampling is $32 \mathrm{~s}$ from the beginning of the observations. CoRoT performs aperture photometry with a fixed mask (see Fig. 6 in Léger et al. 2009). The contaminating flux from background stars falling inside the mask amounts to a maximum of 0.9 percent and produces a dilution of the photometric variation of CoRoT-7 lower than $1.8 \times 10^{-4}$ mag that can be safely ignored for our purposes (see Léger et al. 2009, for upper limits on the background flux variations). The flux inside the star's mask is split along detector column boundaries into broad-band red, green, and blue channels.

The observations and data processing are described by Léger et al. (2009), to whom we refer the reader for details. The reduction pipeline applies corrections for the sky background and the pointing jitter of the satellite, which is particularly relevant during ingress and egress from the Earth shadow. Measurements during the crossing of the South Atlantic Anomaly of the Earth's magnetic field, which amounts to about 7-9 percent of each satellite orbit, are discarded. High-frequency spikes due to cosmic ray hits and outliers are removed by applying a 7-point running mean. The final duty cycle of the $32-\mathrm{s} \mathrm{ob-}$ servations is 88.8 percent for the so-called N2 data time series that are accessible through the CoRoT Public Data Archive at the IAS (http://idoc-corot.ias.u-psud.fr/). To increase the signal-to-noise ratio and reduce systematic drifts present in individual channels, we summed up the flux in the red, green, and blue channels to obtain a lightcurve in a spectral range extending from 300 to $1100 \mathrm{~nm}$. More information on the instrument, its operation, and performance can be found in Auvergne et al. (2009).

The transits are removed using the ephemeris of Léger et al. (2009) and the out-of-transit data are binned by computing average flux values along each orbital period of the satellite (6184 s). This has the advantage of removing tiny systematic variations associated with the orbital motion of the satellite (cf. Alonso et al. 2008; Auvergne et al. 2009). In such a way we obtain a lightcurve consisting of 1793 average points ranging from HJD 2454398.0719 to HJD 2454528.8877 , i.e., with a duration of 130.8152 days. The average standard deviation of the points is $2.678 \times 10^{-4}$ in relative flux units. The maximum observed flux in the average point time series at HJD 2454428.4232 is adopted as a reference unit level corresponding to the unspotted star, since the true value of the unspotted flux is unknown.

\section{Lightcurve and radial velocity modelling}

\subsection{Spot modelling of wide-band lightcurves}

The reconstruction of the surface brightness distribution from the rotational modulation of the stellar flux is an ill-posed problem, because the variation in the flux vs. rotational phase only contains information on the distribution of the brightness inhomogeneities vs. longitude. The integration over the stellar disc effectively cancels any latitudinal information, particularly when the inclination of the rotation axis along the line of sight is close to $90^{\circ}$, as assumed in the present case (see Sect. 4 and Lanza et al. 2009a). Therefore, we need to include a priori information in the lightcurve inversion process to obtain a unique and stable map. This is done by computing a maximum entropy (hereafter ME) map, which has been proven to successfully reproduce active region distribution and area variations in the case of the Sun (cf. Lanza et al. 2007).

In our model, the star is subdivided into 200 surface elements, namely 200 squares with sides of $18^{\circ}$, with each element containing unperturbed photosphere, dark spots, and facular areas. The fraction of an element covered by dark spots is indicated by the filling factor $f$, the fractional area of the faculae is $Q f$, and the fractional area of the unperturbed photosphere is $1-(Q+1) f$. The contribution to the stellar flux coming from the $k$ th surface element at the time $t_{j}$, where $j=1, \ldots, N$, is an index numbering the $N$ points along the lightcurve, is given by

$$
\begin{aligned}
\Delta F_{k j}= & I_{0}\left(\mu_{k j}\right)\left\{1-(Q+1) f+c_{\mathrm{s}} f\right. \\
& \left.+Q f\left[1+c_{\mathrm{f}}\left(1-\mu_{k j}\right)\right]\right\} A_{k} \mu_{k j} w\left(\mu_{k j}\right),
\end{aligned}
$$

where $I_{0}$ is the specific intensity in the continuum of the unperturbed photosphere at the isophotal wavelength of the observations, $c_{\mathrm{s}}$ and $c_{\mathrm{f}}$ are the spot and facular contrasts (cf. Lanza et al. 2004), $A_{k}$ is the area of the $k$ th surface element,

$w\left(\mu_{k j}\right)= \begin{cases}1 & \text { if } \mu_{k j} \geq 0 \\ 0 & \text { if } \mu_{k j}<0\end{cases}$

is its visibility, and

$\mu_{k j} \equiv \cos \psi_{k j}=\sin i \sin \theta_{k} \cos \left[\ell_{k}+\Omega\left(t_{j}-t_{0}\right)\right]+\cos i \cos \theta_{k}$

is the cosine of the angle $\psi_{k j}$ between the normal to the surface element and the direction of the observer, with $i$ the inclination of the stellar rotation axis along the line of sight, $\theta_{k}$ the colatitude, and $\ell_{k}$ the longitude of the $k$ th surface element; $\Omega$ denotes the angular velocity of rotation of the $\operatorname{star}\left(\Omega \equiv 2 \pi / P_{\text {rot }}\right.$, with $P_{\text {rot }}$ the stellar rotation period), and $t_{0}$ the initial time. The specific intensity in the continuum varies according to a quadratic limbdarkening law, as adopted by Lanza et al. (2003) for the case of the Sun, viz. $I_{0} \propto a_{\mathrm{p}}+b_{\mathrm{p}} \mu+c_{\mathrm{p}} \mu^{2}$. The stellar flux computed at the time $t_{j}$ is then $F\left(t_{j}\right)=\sum_{k} \Delta F_{k j}$. To warrant a relative precision of the order of $10^{-5}$ in the computation of the flux $F$, each surface element is further subdivided into $1^{\circ} \times 1^{\circ}$-elements and their contributions, calculated according to Eq. (1), are summed up at each given time to compute the contribution of the $18^{\circ} \times$ $18^{\circ}$ surface element to which they belong.

We fit the lightcurve by varying the value of $f$ over the surface of the star, while $Q$ is held constant. Even after we fix the rotation period, the inclination, and the spot and facular contrasts (see Lanza et al. 2007, for details), the model has 200 free parameters and suffers from non-uniqueness and instability. To find a unique and stable spot map, we apply ME regularization, as described in Lanza et al. (2007), by minimizing a functional $Z$, which is a linear combination of the $\chi^{2}$ and the entropy functional $S$; i.e.,

$Z=\chi^{2}(\boldsymbol{f})-\eta S(\boldsymbol{f})$

where $f$ is the vector of the filling factors of the surface elements, $\eta>0$ a Lagrangian multiplier determining the tradeoff between lightcurve fitting and regularization. The expression 
for $S$ is given in Lanza et al. (1998). The entropy functional $S$ is constructed in such a way that it attains its maximum value when the star is free of spots. Therefore, by increasing the Lagrangian multiplier $\eta$, we increase the weight of $S$ in the model and the area of the spots is progressively reduced. This gives rise to systematically negative residuals between the observations and the best-fit model when $\eta>0$. The optimal value of $\eta$ depends on the information content of the lightcurve, which in turn depends on the ratio of the amplitude of its rotational modulation to the average standard deviation of its points. In the case of CoRoT-7, the amplitude of the rotational modulation is $\sim 0.018$, while the average standard deviation of the points is $\sim 2.7 \times 10^{-4}$ in relative flux units, giving a signal-to-noise ratio of $\sim 65-70$. This is sufficient to adopt the same regularization criterion applied in the case of CoRoT-2 (see Lanza et al. 2009a), iterating the value of $\eta$ until the condition $\left|\mu_{\text {reg }}\right| \simeq \epsilon_{0}$ is met, where $\mu_{\text {reg }}$ is the mean of the residuals, and $\epsilon_{0} \equiv \sigma_{0} / \sqrt{N}$ their standard error, i.e., the ratio of their standard deviation $\sigma_{0}$ in the case of the unregularized best fit (i.e., for $\eta=0$ ) to the square root of the number $N$ of points in each fitted subset of the lightcurve having a duration $\Delta t_{\mathrm{f}}$ (see below).

In the case of the Sun, by assuming a fixed distribution of the filling factor, it is possible to obtain a good fit of the irradiance changes only for a limited time interval $\Delta t_{\mathrm{f}}$, not exceeding 14 days, which is the lifetime of the largest sunspot groups dominating the irradiance variation. For other active stars, the value of $\Delta t_{\mathrm{f}}$ must be determined from the observations themselves, looking for the longest time interval that allows a good fit with the applied model (see Sect. 4).

The optimal values of the spot and facular contrasts and of the facular-to-spotted area ratio $Q$ in stellar active regions are unknown a priori. In our model the facular contrast $c_{\mathrm{f}}$ and the parameter $Q$ enter as the product $c_{\mathrm{f}} Q$, so we can fix $c_{\mathrm{f}}$ and vary $Q$, estimating its best value by minimizing the $\chi^{2}$ of the model, as shown in Sect. 4. Since there are many free parameters in the ME model, for this specific application we make use of the model of Lanza et al. (2003), which fits the lightcurve by assuming only three active regions to model the rotational modulation of the flux plus a uniformly distributed background to account for the variations in the mean light level. This procedure is the same as was used to fix the value of $Q$ in the cases of CoRoT-2 and CoRoT-4 (cf. Lanza et al. 2009a,b).

We assume an inclination of the rotation axis of CoRoT-7 of $i \simeq 80^{\circ}$ (see Sect. 4). Since the information on spot latitude that can be extracted from the rotational modulation of the flux for such a high inclination is negligible, the ME regularization virtually puts all the spots at the sub-observer latitude (i.e., $90^{\circ}-i \approx 10^{\circ}$ ) to minimize their area and maximize the entropy. Therefore, we are limited to mapping only the distribution of the active regions vs. longitude, which can be done with a resolution higher than $\sim 50^{\circ}$ (cf. Lanza et al. 2007, 2009a). Our ignorance of the true facular contribution may lead to systematic errors in the active region longitudes derived by our model because faculae produce an increase in the flux when they are close to the limb, leading to a systematic shift in the longitudes of the active regions used to reproduce the observed flux modulation, as discussed by Lanza et al. (2007) for the Sun and illustrated by Lanza et al. (2009a, cf. Figs. 4 and 5) for CoRoT-2.

\subsection{Activity-induced radial velocity variations}

Surface magnetic fields in late-type stars produce brightness and convection inhomogeneities that shift and distort their spectral line profiles leading to apparent RV variations (cf., e.g., Saar \& Donahue 1997; Saar et al. 1998; Huber et al. 2009). To compute the apparent RV variations induced by stellar active regions, we adopt a simple model for each line profile considering the residual profile $R(\lambda)$ at a wavelength $\lambda$ along the line, i.e.: $R(\lambda) \equiv\left[1-I(\lambda) / I_{\mathrm{c}}\right]$, where $I(\lambda)$ is the specific intensity at wavelength $\lambda$ and $I_{\mathrm{c}}$ the intensity in the continuum adjacent to the line. The local residual profile is assumed to have a Gaussian shape with thermal and microturbulent width $\Delta \lambda_{\mathrm{D}}$ (cf., e.g., Gray 1988, Ch. 1); i.e.,

$R(\lambda) \propto \exp \left[-\left(\frac{\lambda-\lambda_{0}}{\Delta \lambda_{\mathrm{D}}}\right)^{2}\right]$,

where $\lambda_{0}$ is the local central wavelength. For simplicity, $R(\lambda)$ is assumed to be independent of the disc position and the presence of spots or faculae, so the local specific intensity along the line $I(\lambda)$ depends only on the variation in the continuum intensity $I_{\mathrm{c}}$ owing to limbdarkening and the effects of dark spots and bright faculae, as specified by Eq. (1).

To include the effects of surface magnetic fields on convective motions, we consider the decrease of macroturbulence velocity and the reduction of convective blueshifts in active regions. Specifically, we assume a local radial-tangential macroturbulence, as introduced by Gray (1988), with a distribution function $\Theta$ for the radial velocity $v$ of the form:

$\Theta(v, \mu)=\frac{1}{2} \frac{\exp \left[-\left(\frac{v}{\zeta_{\mathrm{RT}} \sqrt{1-\mu^{2}}}\right)^{2}\right]}{\sqrt{\pi} \zeta_{\mathrm{RT}} \sqrt{1-\mu^{2}}}+\frac{1}{2} \frac{\exp \left[-\left(\frac{v}{\zeta_{\mathrm{RT}}}\right)^{2}\right]}{\sqrt{\pi} \zeta_{\mathrm{RT}} \mu}$,

where $\zeta_{\mathrm{RT}}$ is the macroturbulence and $\mu$ is given by Eq. (3). The profile emerging from each surface element is obtained by convolving the local profile in Eq. (5) with the Doppler shift distribution as generated by the macroturbulence function in Eq. (6). The parameter $\zeta_{\mathrm{RT}}$ is assumed to be reduced in spotted and facular areas, according to their total filling factor, i.e., $\zeta_{\mathrm{RT}}=\zeta_{0}[1-(Q+1) f]$, where $\zeta_{0}$ is the unperturbed macroturbulence. This is a convenient parameterization of the quenching effect of surface magnetic fields on convective turbulence, at least in the framework of our simplified model, and is supported by the observations of the reduction of turbulent velocity fields in the plage regions of the Sun (cf., e.g., Title et al. 1992).

Convective blueshifts arise from the fact that in stellar photospheres most of the flux comes from the extended updrafts at the centre of convective granules. At the centre of the stellar disc, the vertical component of the convective velocity produces a maximum blueshift, while the effect vanishes at the limb where the projected velocity is zero. The cores of the deepest spectral lines form in the upper layers of the photosphere where the vertical convective velocity is low, while the cores of shallow lines form in deeper layers with higher vertical velocity. Therefore, the cores of shallow lines are blueshifted with respect to the cores of the deepest lines. Gray (2009) shows this effect by plotting the endpoints of line bisectors of shallow and deep lines on the same velocity scale. He shows that the amplitude of the relative blueshifts scales with the spectral type and the luminosity class of the star. For the G8V star $\tau$ Ceti, which has a spectral type close to the G9V of CoRoT-7, the convective blueshifts should be similar to those of the Sun, so we adopt solar values in our simulations. In active regions, vertical convective motions are quenched, so we observe an apparent redshift of the spectral lines in spotted and facular areas in comparison to the unperturbed photosphere. Meunier et al. (2010) quantify this effect in 
the case of the Sun, and we adopt their results, considering an apparent redshift $\Delta V_{\mathrm{f}}=200 \mathrm{~m} \mathrm{~s}^{-1}$ in faculae and $\Delta V_{\mathrm{s}}=300 \mathrm{~m} \mathrm{~s}^{-1}$ in cool spots.

In principle, the integrated effect of convective redshifts can be measured in a star by comparing RV measurements obtained with two different line masks, one including the shallow lines and the other the deep lines (cf. Meunier et al. 2010). For CoRoT-7, which lacks such measurements, we apply the results of Gray (2009) and adopt solar-like values as the best approximation.

Considering solar convection as a template, intense downdrafts are localized in the dark lanes at the boundaries of the upwelling granules, but they contribute a significantly smaller flux because of their lower brightness and smaller area. While a consideration of those downdrafts is needed to simulate the shapes of line bisectors, it is beyond the scope of our simplified approach that assumes that the whole profile of our template line forms at the same depth inside the photosphere. Therefore, we restrict our model to the mean apparent RV variations by neglecting the associated variations of the bisector shape and do not include the effect of convective downdrafts, as well as those of other surface flows typical of solar active regions, such as the Evershed effect in sunspots (cf., e.g., Meunier et al. 2010).

The local central wavelength $\lambda_{0}$ of the $k$ th surface element at time $t_{j}$ is given by $\lambda_{0 k j}=\lambda_{\mathrm{R}}\left(1+v_{k j} / c\right)$, where $v_{k j}$ is its radial velocity,

$v_{k j}=-(v \sin i) \sin \theta_{k} \sin \left[\ell_{k}+\Omega\left(t_{j}-t_{0}\right)\right]+\Delta V_{\mathrm{cs}}$,

with $c$ the speed of light, $v \sin i$ the stellar projected rotational velocity, $\lambda_{\mathrm{R}}$ the rest wavelength of the spectral line, and $\Delta V_{\mathrm{cs}}$ the apparent convective redshift arising from the reduction of convective blueshifts in spots and faculae, which is parameterized as

$\Delta V_{\mathrm{cs}}=\left(\Delta V_{\mathrm{s}}+Q \Delta V_{\mathrm{f}}\right) f_{k} \mu_{k j}$,

where $f_{k}$ is the spot filling factor of the element, $Q$ the facularto-spotted area ratio, and $\mu_{k j}$ is given by Eq. (3).

We integrate the line specific intensity at a given wavelength over the disc of the star using a subdivision into $1^{\circ} \times 1^{\circ}$ surface elements to obtain the flux along the line profile $F\left(\lambda, t_{j}\right)$ at a given time $t_{j}$. To find the apparent stellar RV, we can fit a Gaussian to the line profile $F\left(\lambda, t_{j}\right)$, or we can determine the centroid of the profile as

$\lambda_{\text {ce }}\left(t_{j}\right)=\frac{\int \lambda \mathcal{R}\left(\lambda, t_{j}\right) \mathrm{d} \lambda}{\int \mathcal{R}\left(\lambda, t_{j}\right) \mathrm{d} \lambda}$,

where $\mathcal{R} \equiv\left[1-F\left(\lambda, t_{j}\right) / F_{\mathrm{c}}\left(t_{j}\right)\right]$ is the residual profile of the spectral line computed from the ratio of the flux in the line $F\left(\lambda, t_{j}\right)$ to the flux $F_{\mathrm{c}}\left(t_{j}\right)$ in the adjacent continuum at any given time $t_{j}$.

A single line profile computed with the above model can be regarded as a cross-correlation function (hereafter $\mathrm{CCF}$ ) obtained by cross-correlating the whole stellar spectrum with a line mask consisting of Dirac delta functions giving the rest wavelength and depth of each individual line (e.g., Queloz et al. 2001). Therefore, to derive the RV from a single synthetic line profile is equivalent to measuring the RV from a CCF, either by fitting it with a Gaussian or by computing its centroid wavelength. A better approach would be to simulate the whole stellar optical spectrum or an extended section of it to account for the wavelength dependence of the spot and facular contrasts, as well as of the convective inhomogeneities (cf., e.g., Desort et al. 2007; Lagrange et al. 2010; Meunier et al. 2010). Again, in view of our limited knowledge of the distribution of active regions on the stellar surface, our simplified approach is adequate for estimating the RV perturbations induced by magnetic fields.

\subsubsection{Some illustrative examples}

To illustrate how active regions affect the measurement of stellar RV, we consider the simple case of a single active region on a slowly rotating star, assuming stellar parameters similar to the cases presented by Saar \& Donahue (1997) and Desort et al. (2007) for comparison purposes. Specifically, we adopt $v \sin i=6.5 \mathrm{~km} \mathrm{~s}^{-1}$ and a macroturbulence $\zeta_{0}=5.5 \mathrm{~km} \mathrm{~s}^{-1}$ to make macroturbulence effects visible. For the moment, we do not include convective redshifts in spots and faculae because they were not taken into account in those simulations. We consider a single active region of square shape $18^{\circ} \times 18^{\circ}$ located at the equator of a star observed equator-on, to maximize the $\mathrm{RV}$ variation. The spot filling factor is assumed to be $f=0.99$ and their contrast $c_{\mathrm{s}}=0.01$, i.e., the spot effective temperature is much lower than the photospheric temperature. When faculae are included, we adopt $c_{\mathrm{f}}=0.115$ and $Q=9$ to make their contribution clearly evident. In Fig. 1 we show the apparent RV and the relative flux variations produced by the active region vs. the rotation phase. The RV variations are computed by fitting a Gaussian to the line profile, as in Saar \& Donahue (1997), while by applying the centroid method we find values that differ by at most 10-15 percent. The greatest apparent variation is obtained in the case of a purely dark spot without any bright facula or reduced macroturbulence. In this case, the line profile integrated over the stellar disc shows a bump at the RV of the spot because the intensity of the local continuum is reduced in the spot (see Fig. 1 in Vogt \& Penrod 1983, for an illustration of the effect). A comparison with the amplitudes calculated with the formulae of Saar \& Donahue (1997) or Desort et al. (2007) shows agreement within $\sim 10$ percent. The RV perturbation is positive and the flux decreases when the spot is on the approaching half of the disc. When the spot transits across the central meridian, the RV perturbation becomes zero and the flux reaches a relative minimum. Finally, the RV perturbation becomes negative when the spot is on the receding half of the disc and the flux is increasing.

Including the radial-tangential macroturbulence produces a decrease in the RV amplitude because macroturbulence is reduced in spots so that the local line profile becomes narrower and deeper, while its equivalent width is not significantly affected (Gray 1988). This reduces the height of the bump in the integrated profile acting in the opposite direction to the perturbation produced by a dark spot, as is evident in the case where only a macroturbulence reduction is included in the model. The wideband flux variations are not affected by including the macroturbulence in any case.

The effect of the faculae is to increase the local continuum intensity when an active region is close to the limb, leading to more local absorption, which produces a dip in the integrated line profile. Since the contrast of the faculae is greatly reduced when they move close to the centre of the disc, this has the effect of narrowing the RV peak produced by an active region when dark spots and faculae are simultaneously present, i.e., faculae increase the frequency of the RV variation. Specifically, the frequency of the RV variation in the case of a single active region is twice the rotation frequency when dark spots or a reduction of the macroturbulence are present (cf. Fig. 1, upper panel, where we see a complete RV oscillation along one transit of the active region that lasts half a stellar rotation). Including solar-like faculae can increase the frequency of the variation up to four times the rotation frequency when facular effects gain in significance (i.e., $c_{\mathrm{f}} Q>1$ ). This is illustrated in the upper panel of Fig. 2 with the RV variation produced by an active region with a 
A. F. Lanza et al.: Activity, rotation, and radial velocity variations of CoRoT-7

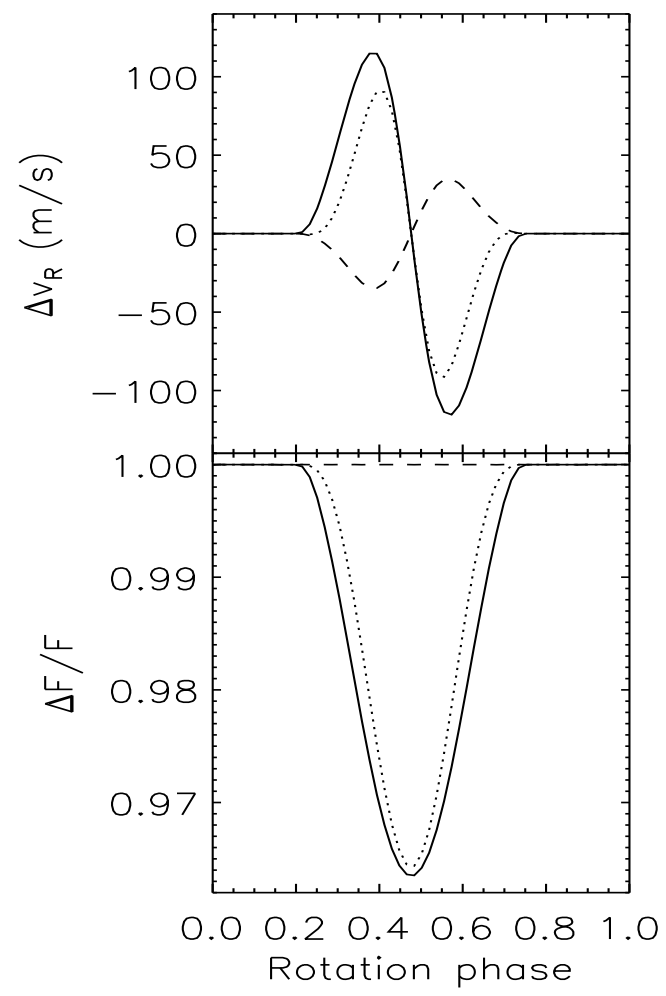

Fig. 1. Apparent RV variation (upper panel) and relative stellar flux (lower panel) vs. the rotation phase produced by a single active region on a star seen equator-on. Different linestyles indicate different kinds of inhomogeneities inside the active region. Dashed: reduced macroturbulence without brightness perturbation; solid: dark spot and reduced macroturbulence; dotted: dark spot, bright facula, and reduced macroturbulence. The flux is measured in units of the unperturbed stellar flux.

sizeable facular contribution (see below) showing two complete oscillations along one transit.

Next we consider the apparent redshifts due to the reduction of convective blueshifts in spots and faculae. To make the effect evident, now we adopt a spot contrast $c_{\mathrm{s}}=0.665$, a facular contrast $c_{\mathrm{f}}=0.115$, and $Q=4.5$. For the convective redshifts, we assume $\Delta V_{\mathrm{s}}=500 \mathrm{~m} \mathrm{~s}^{-1}$ and $\Delta V_{\mathrm{f}}=300 \mathrm{~m} \mathrm{~s}^{-1}$. A lower spot contrast, e.g., $c_{\mathrm{s}}=0.01$, as in the previous simulations, makes the contribution of the local redshifted line profile coming from the spotted area very small, reducing the corresponding RV perturbation. In Fig. 2 (upper panel), we plot the RV variation produced by the single active region previously considered, with all the other parameters kept at the previous values. For the purpose of comparison, we also plot the RV perturbation obtained without convective redshifts. In this case, the effects of cool spots and bright faculae almost balance each other out because of the higher spot contrast, and the oscillation of the RV shows a high frequency. On the other hand, including convective redshifts brings the frequency of the RV variation close to the rotation frequency, with a single maximum per rotation. This happens because the perturbation is always positive, while all the other effects change their sign when the active region transits from the approaching to the receding half of the stellar disc. Moreover, the convective redshifts do not depend per se on the contrast factors $c_{\mathrm{s}}$ and $c_{\mathrm{f}}$, and the shift due to the faculae is amplified by the facular-to-spotted area ratio $Q$. The modulation of the continuum flux is of course not affected by the convective shifts, so we obtain the same lightcurve independent of the value of $\Delta V_{\mathrm{s}}$ and $\Delta V_{\mathrm{f}}$ (cf. Fig. 2, lower panel).

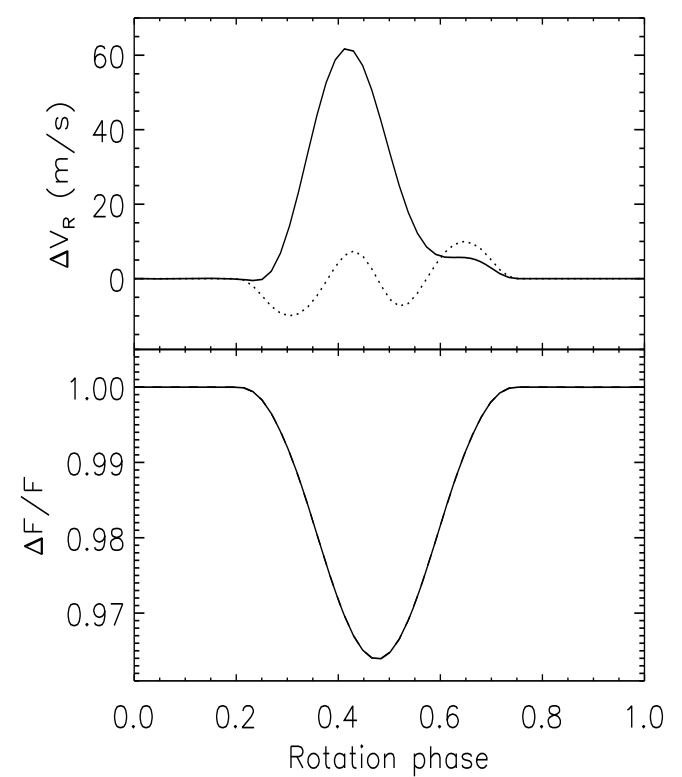

Fig. 2. Same as Fig. 1 when including (solid line) and not including (dotted line) the convective redshifts in cool spots and bright faculae in the simulation (see the text for the active region parameters).

Particularly for not too dark spots (i.e., $c_{\mathrm{s}} \gtrsim 0.4$ ) and small $v \sin i$, convective redshifts cannot be neglected, therefore, in the simulation of the activity-induced RV perturbations in solar-like stars, as pointed out by Meunier et al. (2010). In the case of the Sun, redshifts not only increase the RV perturbation up to an order of magnitude, but also bring its dominant period close to the solar rotation period, while without including them those authors find that the dominant period corresponds to the first harmonic of the solar rotation period.

The amplitude of the RV variation produced by an active region depends on several parameters that are poorly known, i.e., the latitude of the active region, the spot and facular contrasts, the convective redshifts, and the macroturbulence parameter that is difficult to disentangle from rotational broadening in a slowly rotating star such as CoRoT-7 (Léger et al. 2009). Moreover, the spot and facular contrasts depend on the wavelength (Lanza et al. 2004), leading to a difference of $\approx 10$ percent in the RV variations as derived from different orders of an echelle spectrum (cf., e.g., Desort et al. 2007), and the convective redshift depends on the formation depth of a spectral line.

The simultaneous presence of several active regions gives rise to a complex line profile distortion for a slowly rotating star because the perturbations of different regions overlap in the line profile owing to the weak rotational broadening. This implies an additional 15-20 percent uncertainty in the determination of the shift of the profile by the Gaussian fit or the centroid method, as found by comparing the RV determinations obtained with the two methods in the case of line profiles simulated with infinite signal-to-noise ratio and perfectly regular sampling. In consideration of all these limitations, the absolute values of the RV variations computed with our model are uncertain by 20-30 percent for complex distributions of active regions, such as those derived by our spot modelling technique as applied to CoRoT-7 lightcurves.

\subsubsection{Radial velocity variations from spot modelling}

We can use the distribution of active regions as derived from our spot modelling to synthesize the corresponding RV variations 
according to the approach outlined in Sect. 3.2. Since our spot model assumes that active regions are stable for the time interval of each fitted time series $\Delta t_{\mathrm{f}}$, the distribution of surface inhomogeneities can be used to synthesize the RV variations having a timescale of $\Delta t_{\mathrm{f}}$ or longer. Active regions with shorter lifetimes produce a photometric modulation that appears in the residuals of the best fit to the lightcurve. As we see in Sect. 5.1, most of the short-term variability occurs on timescales of 4-5 days, i.e., significantly shorter than the rotation period of CoRoT-7, so we can neglect, as a first approximation, the variation in the disc position of active regions due to stellar rotation and estimate their area as if they were located at the centre of the disc. Specifically, we first express the residuals as the relative deviation $\Delta F / F_{0}$ between the observed flux and its best fit measured in units of the reference unspotted flux $F_{0}$. Then we subtract its mean value $\mu_{\text {res }} \equiv\left\langle\Delta F / F_{0}\right\rangle$ from $\Delta F / F_{0}$ because the mean value corresponds to a uniformly distributed population of active regions that do not produce any RV variation. At each given time $t$, we adopt the relative deviation $\left|\Delta F / F_{0}-\mu_{\text {res }}\right|$ as a measure of the filling factor of the active regions producing the short-term $\mathrm{RV}$ variations. In doing so, we neglect limb-darkening effects and assume that those active regions consist of completely dark spots $\left(c_{\mathrm{s}}=0\right)$. Finally, we compute the RV perturbation due to such brightness inhomogeneities by means of Eq. (1) of Desort et al. (2007) obtaining

$\Delta V_{\mathrm{Ri}}=800\left(\frac{\Delta F}{F}-\mu_{\mathrm{res}}\right)(v \sin i)^{1.1}$,

where the RV perturbation is measured in $\mathrm{m} \mathrm{s}^{-1}$ and the $v \sin i$ of the star in $\mathrm{km} \mathrm{s}^{-1}$. For simplicity, Eq. (10) assumes that the perturbation has the same sign as $\Delta F / F_{0}-\mu_{\text {res }}$, which should produce statistically correct results for a sufficiently long time series, i.e., with equally probable positive and negative deviations. In our case, Eq. (10) gives an upper limit for the amplitude of the RV perturbation because it assumes that an active region spans the diameter of the stellar disc. This is appropriate because we want to assess the impact of short-lived active regions on the detection of exoplanets. On the other hand, the effects of convective redshifts in short-lived active regions is estimated as

$\Delta V_{\mathrm{Rc}}=\left(\Delta V_{\mathrm{s}}+Q \Delta V_{\mathrm{f}}\right)\left(\frac{\Delta F}{F}-\mu_{\mathrm{res}}\right)$,

where the sign of $\Delta V_{\mathrm{Rc}}$ has been chosen to maximize the total short-term RV perturbation when added to that of Eq. (10). By adding both kinds of short-term perturbations to the longterm RV modulation produced by the active regions mapped by the spot modelling, we compute the total synthetic RV variation induced by stellar activity.

\section{Model parameters}

The fundamental stellar parameters are taken from Léger et al. (2009) and are listed in Table 1. The limbdarkening parameters $a_{\mathrm{p}}, b_{\mathrm{p}}$, and $c_{\mathrm{p}}$ (cf. Sect. 3.1) have been derived from Kurucz (2000) model atmospheres for $T_{\text {eff }}=5275 \mathrm{~K}, \log g=$ $4.50\left(\mathrm{~cm} \mathrm{~s}^{-2}\right)$ and solar abundances, by adopting the CoRoT white-band transmission profile given by Auvergne et al. (2009). Recently, Bruntt et al. (2010) have slightly revised stellar parameters, but adopting their values does not produce any significant modification in our modelling.

The rotation period adopted for our spot modelling has been derived from a periodogram analysis of the lightcurve giving
Table 1. Parameters adopted for the lightcurve and RV modelling of CoRoT-7.

\begin{tabular}{lrr}
\hline \hline Parameter & & Ref. \\
\hline Star Mass $\left(M_{\odot}\right)$ & 0.93 & L09 \\
Star Radius $\left(R_{\odot}\right)$ & 0.87 & L09 \\
$T_{\text {eff }}(\mathrm{K})$ & 5275 & L09 \\
$\log g\left(\mathrm{~cm} \mathrm{~s}^{-2}\right)$ & 4.50 & L09 \\
$a_{\mathrm{p}}$ & 0.284 & La10 \\
$b_{\mathrm{p}}$ & 0.961 & La10 \\
$c_{\mathrm{p}}$ & -0.254 & La10 \\
$P_{\text {rot }}($ days $)$ & 23.64 & La10 \\
$\epsilon$ & $8.54 \times 10^{-6}$ & La10 \\
Inclination $(\mathrm{deg})$ & 80.1 & L09 \\
$c_{\mathrm{s}}$ & 0.577 & La10 \\
$c_{\mathrm{f}}$ & 0.115 & $\mathrm{~L} 04$ \\
$Q$ & 1.0 & $\mathrm{La} 10$ \\
$\Delta t_{\mathrm{f}}($ days $)$ & $18.688,14.532$ & La10 \\
$v \sin i\left(\mathrm{~km} \mathrm{~s}^{-1}\right)$ & 1.7 & Br10 \\
$\Delta \lambda_{\mathrm{D}}\left(\mathrm{km} \mathrm{s}^{-1}\right)$ & 2.33 & La10 \\
$\zeta_{0}\left(\mathrm{~km} \mathrm{~s}^{-1}\right)$ & 1.2 & Br10 \\
$\Delta V_{\mathrm{s}}\left(\mathrm{m} \mathrm{s}^{-1}\right)$ & 300 & $\mathrm{G} 09, \mathrm{Me} 10$ \\
$\Delta V_{\mathrm{f}}\left(\mathrm{m} \mathrm{s}^{-1}\right)$ & 200 & $\mathrm{G} 09, \mathrm{Me} 10$ \\
\hline
\end{tabular}

References. Br10: Bruntt et al. (2010); G09: Gray (2009); L04: Lanza et al. (2004); L09: Léger et al. (2009); La10: present study; Me10: Meunier et al. (2010).

$P_{\text {rot }}=23.64 \pm 3.62$ days. The uncertainty of this period determination is derived from the total extension of the time series and represents an upper limit. As seen below, our spot model shows that the starspots have a remarkable differential rotation which contributes to an uncertainty of the stellar rotation period of $\sim 6$ percent, i.e., of \pm 1.42 days (cf. Sect. 5.2).

The polar flattening of the star because of the centrifugal potential is computed in the Roche approximation with a rotation period of 23.64 days. The relative difference between the equatorial and the polar radii $\epsilon$ is $8.54 \times 10^{-6}$, which induces a completely negligible relative flux variation of $\approx 10^{-7}$ for a spot coverage of $\sim 2$ percent as a consequence of the gravity darkening of the equatorial region of the star.

The inclination of the stellar rotation axis is impossible to constrain through the observation of the Rossiter-McLaughlin effect owing to the very small planet relative radius $R_{\mathrm{p}} / R_{\text {star }}=$ $0.0187 \pm 3.0 \times 10^{-4}$, the small rotational broadening of the star $v \sin i<3.5 \mathrm{~km} \mathrm{~s}^{-1}$, and its intrinsic line profile variations due to stellar activity (Queloz et al. 2009). Nevertheless, we assume that the stellar rotation axis is orthogonal to the orbital plane of the transiting planet, i.e., with an inclination of $80^{\circ} .1 \pm 0.3$ from the line of sight (cf. Léger et al. 2009).

The maximum time interval that our model can accurately fit with a fixed distribution of active regions $\Delta t_{\mathrm{f}}$ was determined by dividing the total interval, $T=130.8152$ days, into $N_{\mathrm{f}}$ equal segments, i.e., $\Delta t_{\mathrm{f}}=T / N_{\mathrm{f}}$, and looking for the minimum value of $N_{\mathrm{f}}$ that allows a good fit of the lightcurve, as measured by the $\chi^{2}$ statistics. We found that for $N_{\mathrm{f}}<7$ the quality of the best fit degraded significantly with respect to $N_{\mathrm{f}} \geq 7$, owing to a substantial evolution of the pattern of surface brightness inhomogeneities. Therefore, we adopt $\Delta t_{\mathrm{f}}=18.688$ days as the maximum time interval to be fitted with a fixed distribution of surface active regions in order to estimate the best value of the parameter $Q$ (see below). This confirms the result of Queloz et al. (2009), who estimate a starspot coherence time of $\sim 18$ days. However, for the spot modelling in Sects. 5.1 and 5.2, we adopt $N_{\mathrm{f}}=9$, corresponding to $\Delta t_{\mathrm{f}}=14.532$ days, which 
provides better time resolution for studying the evolution of the spot pattern during the intervals with faster modifications.

To compute the spot contrast, we adopt the same mean temperature difference as derived for sunspot groups from their bolometric contrast, i.e., $560 \mathrm{~K}$ (Chapman et al. 1994). In other words, we assume a spot effective temperature of $4715 \mathrm{~K}$, yielding a contrast $c_{\mathrm{s}}=0.577$ in the CoRoT passband (cf. Lanza et al. 2007). A different spot contrast changes the absolute spot coverages, but does not significantly affect their longitudes and their time evolution, as discussed in detail by Lanza et al. (2009a). The facular contrast is assumed to be solar-like with $c_{\mathrm{f}}=0.115$ (Lanza et al. 2004).

The best value of the area ratio $Q$ of the faculae to the spots in each active region was estimated by means of the model of Lanza et al. (2003, cf. Sect. 3.1). In Fig. 3, we plot the ratio $\chi^{2} / \chi_{\min }^{2}$ of the total $\chi^{2}$ of the composite best fit of the entire time series to its minimum value $\chi_{\min }^{2}$, versus $Q$, and indicate the 95 percent confidence level as derived from the F-statistics (e.g., Lampton et al. 1976). After choosing $\Delta t_{\mathrm{f}}=18.688$ days, we fit the rotational modulation of the active regions for the longest time interval during which they remain stable, modelling both the flux increase from the facular component when an active region is close to the limb, as well as the flux decrease from the dark spots when the same region transits across the central meridian of the disc. In such a way, a measure of the relative facular and spot contributions can be obtained, leading to a fairly accurate estimate of $Q$.

The best value of $Q$ turns out to be $Q=1.0$, with an acceptable range extending from $\sim 0$ to $\sim 4$. There is also a small interval of formally acceptable values between 6.0 and 6.5 , but we regard it as a spurious outcome of the $\chi^{2}$ statistical fluctuations. Although a sizeable facular contribution cannot be excluded on the basis of the photometric best fit, we find in Sect. 5.3 that $Q \leq 4$ is required to reproduce the amplitude of the observed $\mathrm{RV}$ variations. The best value of $Q$ for the Sun is 9 (Lanza et al. 2007). Thus our result indicates a lower relative contribution of the faculae to the light variation of CoRoT-7 than in the solar case. The amplitude of the rotational modulation of the star was $\sim 0.0185$ mag during CoRoT observations and $\sim 0.03$ mag during the campaign organized by Queloz et al. (2009), i.e., 6-10 times that of the Sun at the maximum of the elevenyear cycle. This indicates that CoRoT-7 is more active than the Sun, which may account for the reduced facular contribution to its light variations, as suggested by Radick et al. (1998) and Lockwood et al. (2007). The ground-based photometry of Queloz et al. (2009) also indicates that cool spots dominate the optical variability of the star since it becomes redder when it is fainter. The use of the chromatic information of the CoRoT lightcurves to estimate the spot and facular contrasts and filling factors is made impossible by our ignorance of the unperturbed stellar flux levels in the different colour channels that are needed to disentangle the flux perturbations due to spots and faculae, respectively. The continuous variations in the observed fluxes do not allow us to fix such reference levels so that we cannot unambiguously attribute a given flux modulation to cool spots or bright faculae. Moreover, the long-term drifts of the fluxes in the individual colour channels complicate the estimate of the flux variations in each of them, making this approach unfeasible.

To compute the RV variations induced by surface inhomogeneities, we assume a line rest wavelength of $600 \mathrm{~nm}$ and a local thermal plus microturbulence broadening $\Delta \lambda_{\mathrm{D}}=2.3 \mathrm{~km} \mathrm{~s}^{-1}$. The $v \sin i=1.7 \pm 0.2 \mathrm{~km} \mathrm{~s}^{-1}$ is estimated from the stellar radius, inclination, and rotation period following Bruntt et al. (2010). The radial-tangential macroturbulence velocity is assumed to be

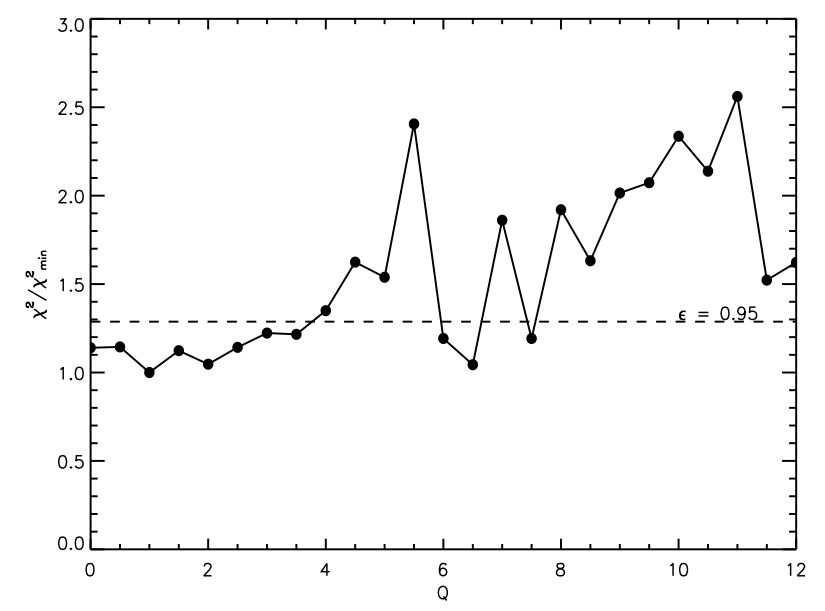

Fig. 3. The ratio of the $\chi^{2}$ of the composite best fit of the entire time series to its minimum value vs. the parameter $Q$, i.e., the ratio of the facular area to the cool spot area in active regions. The horizontal dashed line indicates the 95 percent confidence level for $\chi^{2} / \chi_{\min }^{2}$, determining the interval of acceptable $Q$ values.

$\zeta_{0}=1.2 \pm 0.8 \mathrm{~km} \mathrm{~s}^{-1}$ after Bruntt et al. (2010). It is very difficult to obtain a good estimate of such a parameter because macroturbulence and rotational broadening are largely degenerate owing to the slow rotation of the star (cf. Léger et al. 2009; Bruntt et al. 2010). The convective redshifts in cool spots and faculae, $\Delta V_{\mathrm{s}}$ and $\Delta V_{\mathrm{f}}$, have been assumed to be similar to those of the Sun, following the discussion in Sect. 3.2.

\section{Results}

\subsection{Lightcurve model}

We applied the model of Sect. 3.1 to the out-of-transit CoRoT-7 lightcurve, considering nine intervals of duration $\Delta t_{\mathrm{f}}=$ 14.532 days. The best fit without regularization $(\eta=0)$ has a mean $\mu_{\mathrm{res}}=3.862 \times 10^{-6}$ and a standard deviation of the residuals $\sigma_{0}=1.444 \times 10^{-4}$ in relative flux units. The Lagrangian multiplier $\eta$ is iteratively adjusted until the mean of the residuals $\mu_{\text {res }}=-1.057 \times 10^{-5} \simeq-\sigma_{0} / \sqrt{N}$, where $N=196$ is the mean number of points in each fitted lightcurve interval $\Delta t_{\mathrm{f}}$. The standard deviation of the residuals of the regularized best fit is $\sigma=1.951 \times 10^{-4}$. The composite best fit to the entire lightcurve is shown in the upper panel of Fig. 4, while the residuals are plotted in the lower panel. They show oscillations with a typical timescale of $\sim 4-5$ days that can be related to the rise and decay of small active regions that cover $\approx 0.1$ percent of the stellar disc, i.e., comparable to a large sunspot group. These small active regions cannot be modelled by our approach because they do not produce a significant rotational flux modulation during the 23.6 days of the stellar rotation period as they move across the disc by only $\approx 60^{\circ}-75^{\circ}$ in longitude. By decreasing the degree of regularization, i.e., the value of $\eta$, we can marginally improve the best fit, but at the cost of introducing several small active regions that wax and wane from one time interval to the next and are badly constrained by the rotational modulation. Nevertheless, the oscillations of the residuals do not disappear completely even for $\eta=0$, indicating that CoRoT-7 has a population of shortlived active regions with typical lifetimes of 4-5 days.

A periodogram of the residuals is plotted in Fig. 5, and its main peaks correspond to periods of 5.12 and 3.78 days, respectively, the latter close to the orbital period of CoRoT-7c of 3.69 days. Fitting a sinusoid with this period, we find a 


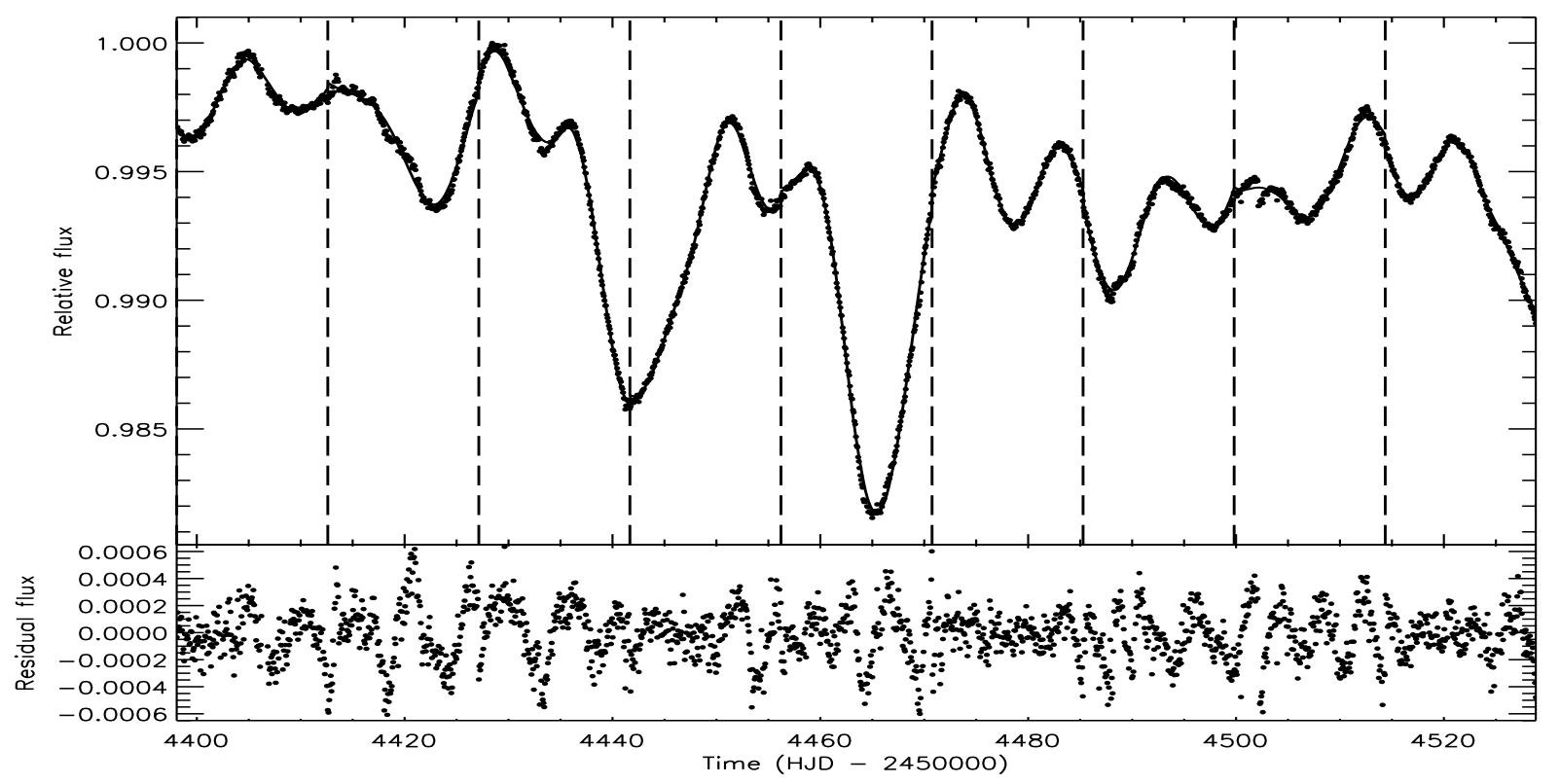

Fig. 4. Upper panel: the out-of-transit lightcurve of CoRoT-7 (dots) and its ME-regularized best fit for a facular-to-spotted area ratio of $Q=1.0$ (solid line). The flux is normalized to the maximum observed flux. The vertical dashed lines mark the individually fitted intervals of $\Delta t_{\mathrm{f}}=$ 14.532 days. Lower panel: the corresponding residuals.

semiamplitude of the light variation of $6.47 \times 10^{-5}$ in relative flux units. The corresponding RV perturbation, estimated with Eqs. (10) and (11), has a semiamplitude of $\sim 0.4 \mathrm{~m} \mathrm{~s}^{-1}$, an order of magnitude less than the oscillation attributed to the planet CoRoT-7c whose semiamplitude is $4.0 \pm 0.5 \mathrm{~m} \mathrm{~s}^{-1}$. Concerning CoRoT-7b, the residual oscillations at its orbital frequency are practically zero.

\subsection{Longitude distribution of active regions and stellar differential rotation}

The distributions of the spotted area vs. longitude are plotted in Fig. 6 for the nine mean epochs of our individual subsets adopting a rotation period of 23.64 days. Longitude zero corresponds to the sub-observer point at the initial epoch, i.e., HJD 2454398.0719. The longitude increases in the same direction as the stellar rotation. This is consistent with the reference frames adopted in our previous studies (Lanza et al. 2009a,b). To allow a comparison of the mapped active regions with the dips in the lightcurve, note that a feature at longitude $\ell$ crosses the central meridian of the star at rotation phase $360^{\circ}-\ell$.

The spot distribution and its evolution suggest three largescale active regions that show different intrinsic longitudinal motions. Assuming that this is caused by differential rotation, we perform linear fits by assuming constant migration rates that are found to be $0.31 \pm 0.12 \mathrm{deg} /$ day for the one fitted with a dotted line; $1.20 \pm 0.19 \mathrm{deg} / \mathrm{day}$ for the one fitted with a dotdashed line; and $0.61 \pm 0.27 \mathrm{deg} / \mathrm{day}$ for the one fitted with a long-dashed line. The relative amplitude of the surface differential rotation, as estimated from the difference between the highest and the lowest migration rates, is $\Delta \Omega / \Omega=0.058 \pm$ 0.017 ; this is only a lower limit since the spot latitudes are unknown. Since CoRoT-7 is more active than the Sun, its active regions may cover a latitude range greater than in the Sun where sunspot groups are confined to $\pm 40^{\circ}$ from the equator (see, e.g., Strassmeier 2009). For the Sun, one finds $\Delta \Omega / \Omega \simeq 0.04-0.05$ by considering active regions confined to within the sunspot belt, i.e., within $\pm\left(35^{\circ}-40^{\circ}\right)$ from the equator. Thus, CoRoT-7 may

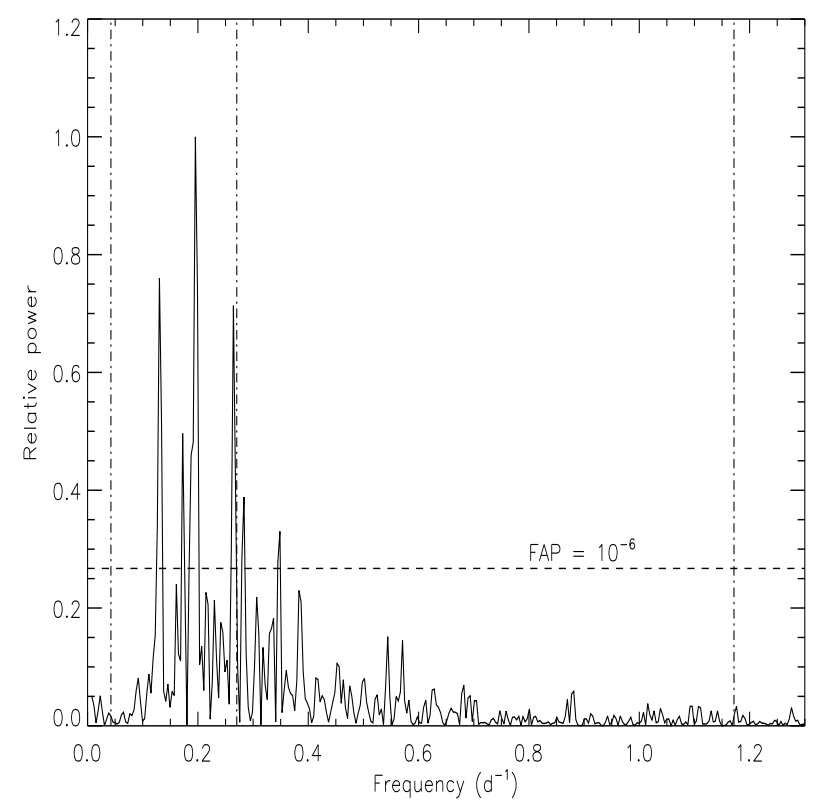

Fig. 5. Lomb-Scargle periodogram of the residual time series of Fig. 4. The dashed horizontal line marks a false-alarm probability of $10^{-6}$. The vertical dot-dashed lines mark the orbital frequencies of the planets CoRoT-7b (right, $P_{\text {orb }}=0.8536$ days) and CoRoT-7c (middle, $P_{\text {orb }}=3.698$ days), as well as the stellar rotation frequency (left, $P_{\text {rot }}=23.64$ days).

have a surface differential rotation comparable to the Sun if its active regions are also mainly localized at low latitudes.

It is interesting to compare the lower limit for the surface shear of CoRoT-7 with the surface differential rotation estimated by Croll et al. (2006) for the K2 dwarf $\epsilon$ Eridani. They find two spots rotating with periods of 11.35 at a latitude of $20^{\circ} .0$ and 11.55 days at 31.5 leading to a differential rotation amplitude of half that of the Sun. This is consistent with a weak dependence of the absolute surface shear $\Delta \Omega$ on the angular velocity $\Omega$, because $\epsilon$ Eri has a rotation period roughly half that of the Sun. Indeed, 
A. F. Lanza et al.: Activity, rotation, and radial velocity variations of CoRoT-7

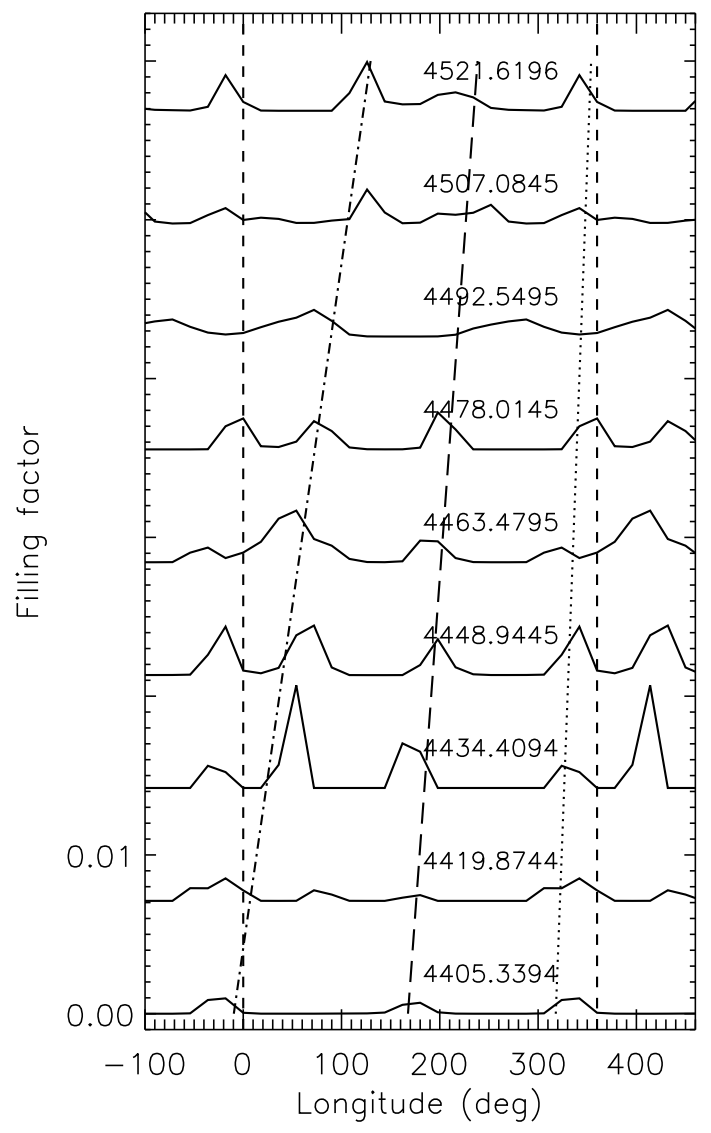

Fig. 6. Distributions of the spotted area vs. longitude during the time intervals centred on the labelled times (HJD - 2450000.0 ), adopting $Q=1.0$. The plots have been vertically shifted to show the apparent migration of individual active regions and repeated beyond $0^{\circ}$ and $360^{\circ}$. The dotted, dot-dashed, and long-dashed lines trace the apparent migration of the three most conspicuous features (see text for details).

Barnes et al. (2005) find $\Delta \Omega \propto \Omega^{0.15}$ for late-type stars at a fixed effective temperature. This suggests that the true pole-equator angular velocity difference in CoRoT-7 may be about a factor of 2-3 greater than observed, if the active regions mapped by our technique are mainly localized at low latitudes.

The stable active longitudes plotted in Fig. 6 show significant area changes on a timescale as short as two weeks, although their overall lifetime may exceed the duration of the lightcurve, i.e., 130 days. As a matter of fact, Queloz et al. (2009) find that the rotational modulation of the optical flux observed during their ground-based campaign from December 2008 to February 2009 matched the extrapolation of the CoRoT lightcurve when a rotation period of 23.64 days was assumed, although 17 complete rotations had elapsed from CoRoT observations. This suggests that the active longitudes may persist for several years, although some large active regions wax and wane on timescales ranging from two weeks (i.e., the time resolution of our mapping) to a few months. The variation in the total spotted area of our surface models vs. time is plotted in Fig. 7 and shows a characteristic timescale of $\sim 60-80$ days. The absolute values of the area per longitude bin and of the total area depend on the spot and facular contrasts adopted for the modelling. Specifically, darker spots lead to a smaller total area while the effect of the facular contrast is more subtle and somewhat influences the longitudinal distribution of the active regions (cf., e.g., Lanza et al. 2009a, Figs. 4 and 5).

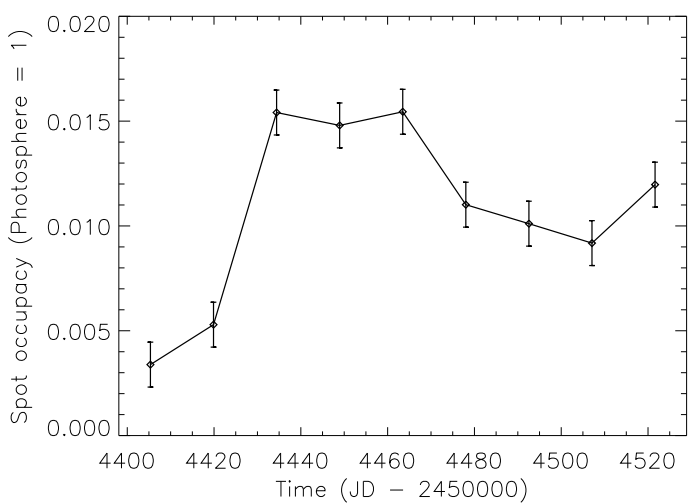

Fig. 7. The total spotted area as derived from our lightcurve model illustrated in Figs. 4 and 6. The uncertainty of the area has been estimated from the errors of the photometric observations.

\subsection{Activity-induced radial velocity variations}

To simulate the apparent RV changes induced by the distribution of active regions derived from our lightcurve modelling, we consider a spectral line with a rest wavelength of $600 \mathrm{~nm}$, i.e., close to the isophotal wavelength of CoRoT observations for which our contrast coefficients are given. The spectral resolution of the line profile is $\lambda / \Delta \lambda=230000$, and 50 profiles are computed per stellar rotation to warrant a good phase sampling. The RV is derived by computing the centroid of each profile, which turns out to be more stable than fitting a Gaussian, which sometimes leads to poor results in the presence of complex line profile distortions. First, we consider the RV perturbation produced by active regions with an evolutionary timescale equal to or greater than $\Delta t_{\mathrm{f}}=14.532$ days as mapped by means of our spot modelling approach for $Q=1$ and $Q=0$ (cool spots only). We plot the simulated RV variations vs. time in the upper panel of Fig. 8 that shows a peak-to-peak amplitude up to $\sim 24.5 \mathrm{~m} \mathrm{~s}^{-1}$ for $Q=1$ and $\sim 20.5 \mathrm{~m} \mathrm{~s}^{-1}$ for $Q=0$. Small discontinuities are seen at the endpoints of each $\Delta t_{\mathrm{f}}$ interval owing to the change in the spot configuration between successive lightcurve fits. Their impact on the simulated RV time series is small and generally comparable to the measurement errors (see below), so it is unnecessary to correct for them.

The observations by Queloz et al. (2009) show a peak-topeak amplitude up to $\sim 40 \mathrm{~m} \mathrm{~s}^{-1}$ for 95 percent of the measurements collected during their campaign from December 2008 to February 2009, while the photometric optical modulation was a factor of $\sim 1.6$ greater. Since the RV perturbation scales approximately linearly with the spot filling factor, our computed amplitude for $Q=1$ agrees with the observed one within $\approx 2-3$ percent, which is a good level of precision for this kind of simulations (cf., e.g., Saar \& Donahue 1997; Desort et al. 2007). Most of the active regions mapped by our spot modelling technique fall within $\pm 20^{\circ}$ from the equator, owing to the preference of ME regularization to put spots close to the sub-observer latitude to minimize their area.

The lower panel of Fig. 8 shows the simultaneous CoRoT white band flux variation. The synthesized fluxes are the same both for models with $(Q=1)$ and without $(Q=0)$ faculae since they must reproduce the rotational modulation as observed by CoRoT. Facular brightening and macroturbulence reduction only play a minor role in the wide-band flux and RV variations, respectively, because $Q=1.0$ and $\zeta_{0}=1.2 \mathrm{~km} \mathrm{~s}^{-1}<v \sin i$ (cf. Sect. 3.2). The dominant effect is the reduction of convective blueshifts in the spotted and facular areas. Since the 


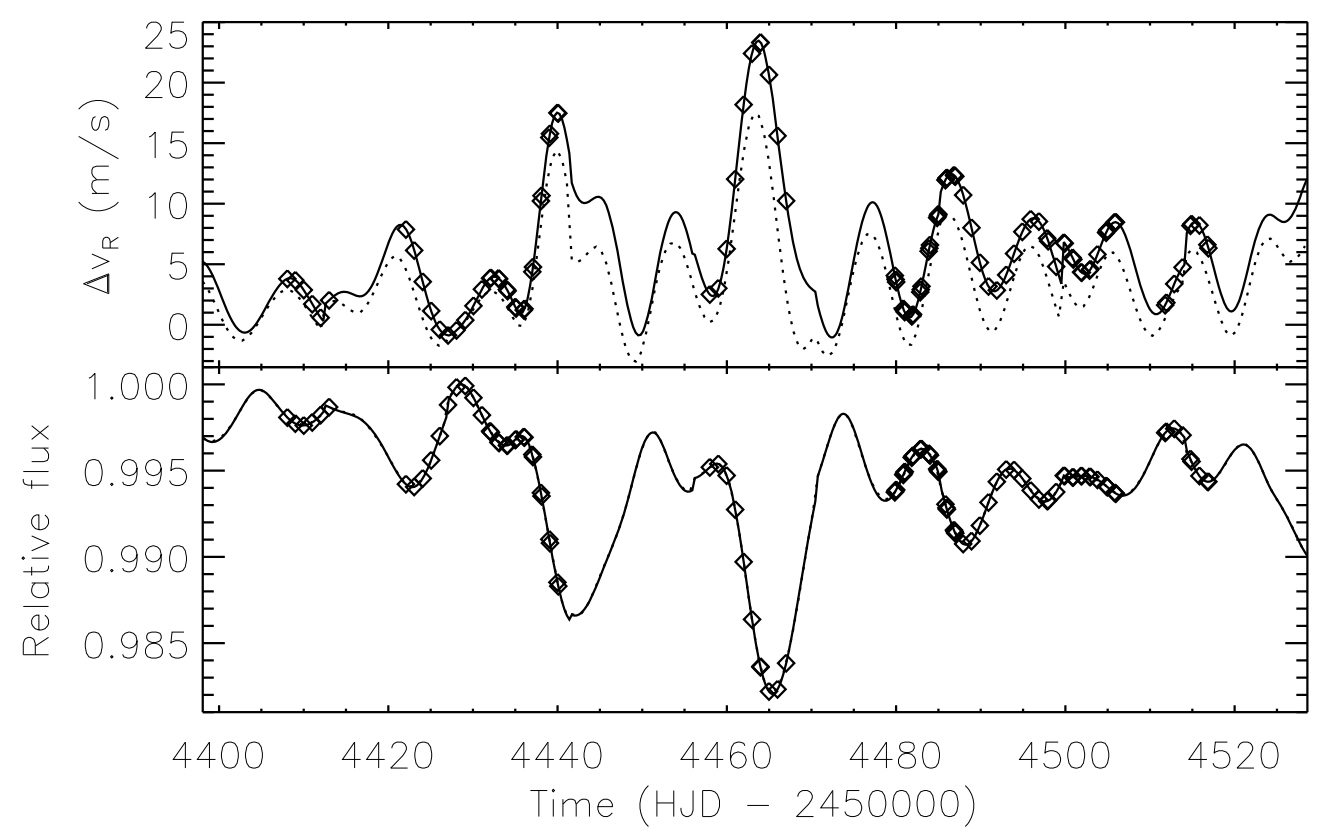

Fig. 8. Upper panel: the synthesized RV variation due to stellar active regions vs. time as derived from our ME spot modelling with $Q=1$ (solid line) and $Q=0$ (dotted line). To illustrate the effect of an examplary ground-based sampling, open diamonds mark the times of HARPS observations by Queloz et al. (2009) after shifting their initial epoch to ten days after the beginning of CoRoT observations. Lower panel: the synthesized relative flux variation in the CoRoT white passband vs. time (solid line) with HARPS time sampling marked by open diamonds.

HARPS measurements by Queloz et al. (2009) were not simultaneous with CoRoT observations, the former have been arbitrarily shifted by ten days with respect to the latter to illustrate the effects of an examplary ground-based time sampling.

To simulate more realistic RV time series, we add the shortterm RV fluctuations associated with the residuals of our composite photometric best fit, computed according to the method outlined in Sect. 3.2.2. To account for HARPS measurement errors, we also add a Gaussian random noise with a standard deviation of $2.0 \mathrm{~m} \mathrm{~s}^{-1}$. In such a way, we consider both the RV variations produced by active regions with a lifetime comparable to $\Delta t_{\mathrm{f}}$ or longer, as mapped by our spot modelling approach, as well as the variations induced by active regions with a shorter lifetime whose photometric effects appear as the residuals of the best fits.

We compute $10^{5} \mathrm{RV}$ time series for $Q=1$, differing by the realization of the Gaussian noise and the initial epoch that is chosen from a uniform random distribution to sample all the parts of the synthetic RV curve in Fig. 8 and the short-term fluctuations. To allow for different initial epochs, we extend the synthetic time series by mirror reflections at their endpoints. We choose to compute $10^{5}$ simulations to have enough statistics to sample the distribution of the RV amplitudes down to a probability of the order of $10^{-4}$.

For each time series, we compute the Lomb periodogram, which gives the squared amplitude of a best-fitted sinusoidal signal as a function of the frequency for an uneven time sampling (Lomb 1976). On the other hand, the amplitudes obtained with the Scargle periodogram are normalized in a different way, as required for the computation of the false-alarm probability, making the comparison of the power level at different frequencies less immediate (Scargle 1982).

The power spectrum obtained by averaging the Lomb periodograms of our $10^{5}$ synthesized time series is plotted in Fig. 9 (solid line). Strong aliases at $1.0 \mathrm{day}^{-1}$ are evident in the power spectrum and are due to the daily cadence of ground-based observations. The most prominent peaks fall at the rotation frequency and its first harmonic, as expected when a few dark spots dominate the RV variations (cf. Sect. 3.2.1 and Figs. 1 and 2). This is mainly due to the convective redshifts in active regions because RV time series computed without their effects have a mean amplitude of only $\sim 18 \mathrm{~m} \mathrm{~s}^{-1}$ and an average power spectrum peaking at the first harmonic with a power $\sim 2.5$ times lower at the rotation frequency. This happens because all the other perturbations change sign when an active region transits from the approaching to the receding part of the stellar disc, while convective redshifts are always positive. At the orbital frequencies of CoRoT-7b and CoRoT-7c, the power is lower by factors of $\sim 3$ and $\sim 4$ relative to the rotation frequency, respectively.

Since the HARPS data are not simultaneous with CoRoT data, we cannot compare this power spectrum with the periodogram of the HARPS RV time series. Nevertheless, it is useful to apply the technique proposed by Queloz et al. (2009, Sect. 4) to filter out RV variations due to stellar activity to see to what extent it is capable of removing the simulated variations associated with stellar surface inhomogeneities.

\subsubsection{Filtering out activity-related variations from the synthetic radial velocity curve}

To fit activity-induced RV variations, we model the radial velocity $v_{\mathrm{R}}\left(t_{j}\right)$ at a given time $t_{j}$ as a linear combination of sinusoids at the rotation frequency $\Omega=2 \pi / P_{\text {rot }}$, its first and second harmonics:

$v_{\mathrm{R}}\left(t_{j}\right)=a_{0}+\sum_{m=1}^{3} b_{m} \cos \left(m \Omega t_{j}\right)+c_{m} \sin \left(m \Omega t_{j}\right)$,

where the free coefficients $a_{0}, b_{m}$, and $c_{m}$ are determined by minimizing the chi square between the model and the observations. Specifically, we apply the normal equation method and 
A. F. Lanza et al.: Activity, rotation, and radial velocity variations of CoRoT-7

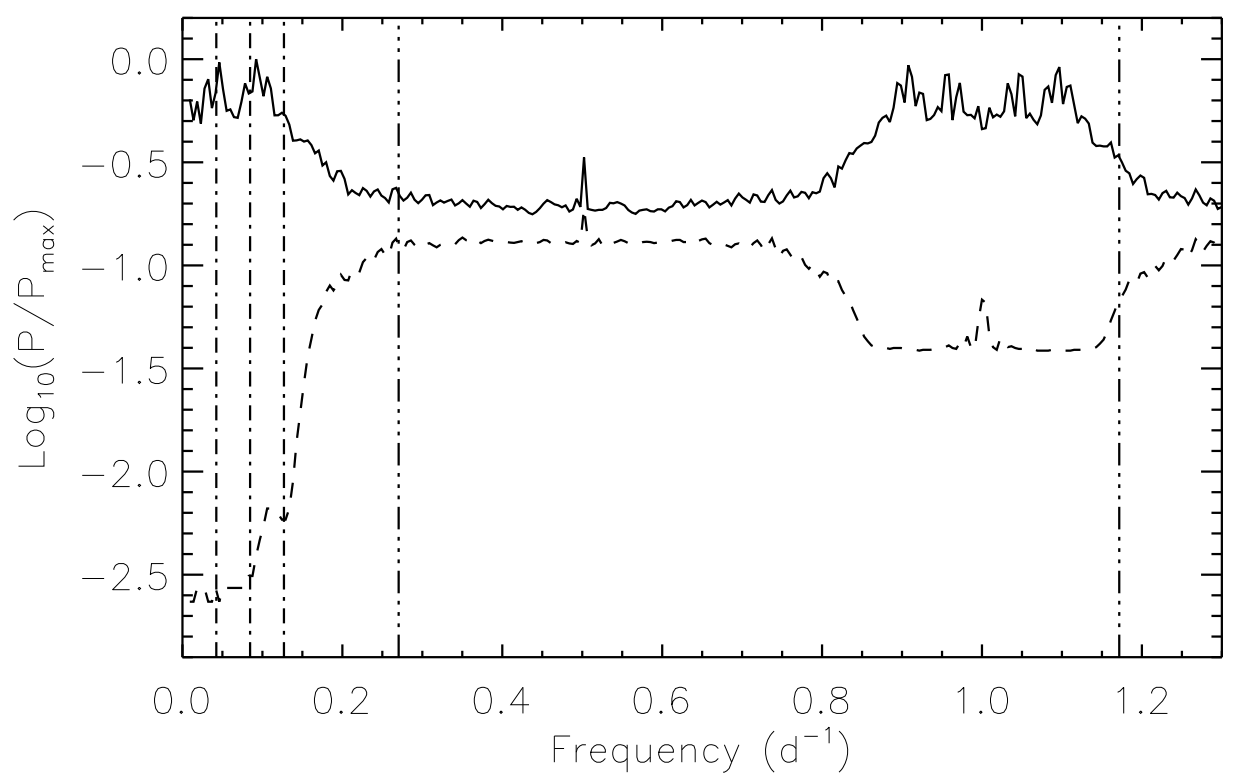

Fig. 9. The average Lomb periodogram of $10^{5}$ synthetic RV time series vs. frequency (solid line) and the average periodogram obtained after filtering the series with the three-harmonic model of Queloz et al. (2009) (dashed line). The power is normalized to the maximum value of the unfiltered mean periodogram. The dot-dashed lines mark the rotation frequency, its first, and second harmonics, while the three-dot-dashed lines mark the orbital frequencies of CoRoT-7c (left) and CoRoT-7b (right), respectively.

use LU decomposition and backsubstitution to solve the corresponding set of linear equations for the model coefficients (Press et al. 1992, Sect. 15.4). As in Queloz et al. (2009), we adopt $P_{\text {rot }}=24.0$ days (although the simulated time series were computed with a model star rotation period of 23.64 days) and apply the three-harmonic fit to subintervals of 20 days. Following Queloz et al. (2009), for each point in the time series several best fits are obtained by scanning the 20-day window through the full data range. These fits are then averaged to compute the best model for that point. Finally, the best-fit time series is subtracted from the initial time series to obtain the filtered series. A Lomb periodogram is computed for each of these filtered series. Figure 9 shows the power spectrum obtained by averaging the periodograms of all the $10^{5}$ residual time series. The power due to stellar activity is reduced by factors of $\sim 4$ and $\sim 2$ at the orbital frequencies of CoRoT-7b and CoRoT-7c, respectively.

To check whether our simulated RV curve of CoRoT-7 contains spurious oscillations at the orbital frequencies of CoRoT-7b or CoRoT-7c, we fit a trigonometric polynomial of the form $B \sin \left(\omega_{\text {orb }} t\right)+C \cos \left(\omega_{\text {orb }} t\right)$ to each residual time series and compute the corresponding semiamplitude $A\left(\omega_{\text {orb }}\right)=\sqrt{B^{2}+C^{2}}$, where $\omega_{\text {orb }}$ is the orbital frequency of the corresponding planet. In the considered $10^{5}$ simulations, $A$ is always lower than $0.85 \mathrm{~m} \mathrm{~s}^{-1}$ at the frequency of CoRoT-7b and lower than $1.7 \mathrm{~m} \mathrm{~s}^{-1}$ at the frequency of CoRoT-7c. The RV oscillations attributed to CoRoT-7b and CoRoT-7c have semiamplitudes $K=$ $3.5 \pm 0.6$ and $K=4.0 \pm 0.5 \mathrm{~m} \mathrm{~s}^{-1}$, respectively. Therefore, the probability that they come from the residual effects of stellar activity is below $10^{-4}$ in both cases.

Since the amplitude of the light modulation during the HARPS observing season was greater by a factor of $\sim 1.6$ than during CoRoT observations, the activity-induced RV oscillations are expected to have been greater during those observations than those simulated from our spot-modelling maps. Nevertheless, even increasing the amplitudes of the residual oscillations by a factor of 2 , the false alarm probability remains below $10^{-4}$.

\subsubsection{Constraining the facular area}

While our photometric modelling does not exclude a facular-tospotted area ratio $Q>1$, we can constrain it on the basis of the observed RV curve. By computing spot models with $Q$ ranging from 4 to 10, we find that the peak-to-peak amplitude of the RV variations increases from 45.7 to $120.3 \mathrm{~m} \mathrm{~s}^{-1}$ for a lightcurve relative amplitude of 0.0185 and spots located within $\pm 20^{\circ}$ from the equator. Given an observed amplitude of $\sim 40 \mathrm{~m} \mathrm{~s}^{-1}$ for a photometric relative modulation of $\sim 0.03$, we conclude that the amplitude of the observed RV curve can be reproduced only if $Q<4$, which is compatible with our adoption of $Q=1$ corresponding to the minimum of the $\chi^{2}$ of the composite photometric best fit. Since the RV amplitude is dominated by convective redshifts in faculae and spots, it could be possible to reduce it for $Q \geq 4$ by reducing $\Delta V_{\mathrm{s}}$ and $\Delta V_{\mathrm{f}}$, or decreasing the spot contrast $c_{\mathrm{S}}$ that leads to smaller spots, hence smaller facular areas for a fixed $Q$. However, the required decrease in $\Delta V_{\mathrm{s}}$ and $\Delta V_{\mathrm{f}}$ should be at least of $\sim 30$ percent for $Q \sim 6$, i.e., significantly greater than allowed by the scaling relationship proposed by Gray (2009). Similarly, the required decrease in $c_{\mathrm{s}}$ should be too large to be compatible with our assumption of active regions with solar-like contrasts.

Our model assumes that the active regions are located close to the equator, according to the ME prescription that tries to minimize their area as much as possible. Nevertheless, we also computed a spot model with $Q=6.5$ and the constraint that active regions are located at high latitudes $\left(\geq 60^{\circ}\right)$, to study the impact on the RV perturbations. The resulting RV time series is very similar, with differences never exceeding $\pm 6 \mathrm{~m} \mathrm{~s}^{-1}$, to the case of low-latitude spots. This happens because the reduction in the convective redshifts, due to projection effects, is compensated by an increase in the continuum flux perturbation due to the faculae, since they are closer to the limb for most of the time. In summary, we conclude that a model with $Q=1$ is appropriate for CoRoT-7, even if its active regions were not constrained to low latitudes. 


\section{Discussion}

We analysed the white band lightcurve of the active late-type star CoRoT-7 that hosts a transiting Earth-like planet while a detailed analysis of its RV variations provided evidence of a second non-transiting Earth-like planet (Léger et al. 2009; Queloz et al. 2009). Since the major source of RV variations in CoRoT-7 is stellar magnetic activity, it needs to be modelled in order to refine the planetary orbital parameters and confirm the presence of the second planet CoRoT-7c (Queloz et al. 2009). In principle, the RV oscillation attributed to CoRoT-7c could be spurious and be the effect of stellar activity because its frequency is close to the fifth harmonic of the stellar rotational frequency. Unfortunately, the CoRoT observations are not simultaneous to the RV time series by Queloz et al. (2009) so our conclusions based on modelling CoRoT data do not constrain, rigorously speaking, magnetic activity properties during the RV observations. Nevertheless, we can derive illustrative conclusions from our results, which can be applied to the analysis of the RV time series.

Our favoured spot model has a facular-to-spotted area ratio $Q=1$, as expected for a star with an activity level significantly higher than the Sun. In our simulated RV time series, most of the power falls at the rotation frequency and its first harmonic. In their analysis of the observed RV time series of CoRoT-7, Queloz et al. (2009) find that most of the power falls at the rotation frequency and at frequencies corresponding to periods of 9.03 and 10.6 days, the latter corresponding to the first harmonic of the rotation frequency. Another prominent peak corresponds to a period of 27.6 days (cf. their Table 2). The period of 27.6 days could be associated with the rotation of spots at high latitudes given that the relative amplitude of the surface differential rotation of CoRoT-7 is at least $\sim 6$ percent. As discussed in Sect. 5.2, a pole-equator angular velocity difference comparable to the Sun, i.e., $\approx 20$ percent, can indeed be present in our star. This is compatible with a rotation period of 23.6 days at the equator and of 27.6 days at high latitudes. The observed peak corresponding to a period of 9.03 days may therefore be the second harmonic of the high-latitude rotation frequency. Recently, Hatzes et al. (private comm.) have suggested that such a modulation may be associated with a third planet. Our approach cannot be applied to support or disprove this hypothesis because our $\mathrm{RV}$ model does not include the effect of surface differential rotation and high-latitude active regions that may be producing the 27.6 day periodicity and its harmonics. Therefore, we have to postpone an investigation of the role of activity-induced effects for the 9.03 day periodicity to a future work.

A large facular component $(Q \geq 4)$ is disfavoured in the case of CoRoT-7 because the amplitude of the RV variation, in this case dominated by convective redshifts in faculae, would then be too large to be compatible with the RV observations.

Finally, in the periodogram of the residuals of our ME composite best fit, there is a significant peak corresponding to a period close to the orbital period of CoRoT-7c (cf. Fig. 5). This peak is also found in the periodogram of the time series of the residuals of the best fit obtained with the method of Queloz et al. (2009), so it is not an artifact associated with the ME regularization. The corresponding RV semiamplitude, estimated with the method described in Sect. 3.2.2, is $\sim 0.4 \mathrm{~m} \mathrm{~s}^{-1}$, i.e., about one order of magnitude less than the RV oscillation produced by the planet, so these fluctuations cannot account for the planetary signature. On the other hand, it cannot be excluded that those photometric oscillations, with a semiamplitude of only $\sim 6 \times 10^{-5} \mathrm{mag}$, could be induced by the interaction of the planet with the stellar coronal magnetic field or by a perturbation of the stellar dynamo by the planet, as conjectured by, e.g., Lanza (2008, 2009).

\section{Conclusions}

We have applied the spot modelling method introduced by Lanza et al. $(2009 \mathrm{a}, \mathrm{b})$ to the lightcurve of the planet-hosting star CoRoT-7. After adopting a facular-to-spotted area ratio $Q=1$, we find evidence of three persistent active longitudes within which individual active regions form and decay with lifetimes ranging from a couple of weeks to a few months. The active longitudes migrate in the adopted reference frame at different rates, which suggests a surface differential rotation with a relative amplitude of at least $0.058 \pm 0.017$. The overall spotted area reaches a maximum of 1.6 percent of the whole surface if the contrasts of dark spots and faculae are adopted as solar-like. The residuals of the best fit indicate a population of small spots with areas of the order of $5 \times 10^{-4}$ of the whole stellar surface that evolve on a typical timescale of $\sim 4-5$ days. Moreover, we find an oscillation of the optical flux with a semiamplitude $\sim 6 \times 10^{-5}$ mag with the orbital period of the planet CoRoT-7c.

We introduce a model to simulate the apparent RV variations induced by magnetic activity that includes the effect of surface brightness inhomogeneities, as well as a reduction in the macroturbulence and convective blueshifts in active regions. We synthesize a time series for the RV variations of CoRoT-7 adopting the spot model obtained from the CoRoT white-band lightcurve and assuming that active regions have solar-like contrasts and are located within $\pm 20^{\circ}$ from the equator. Unfortunately, HARPS and CoRoT observations are not simultaneous, so any model based on the latter does not rigorously constrain the observed RV variations. Nevertheless, an extrapolation of our model matches the observed RV amplitude.

We fit the RV time series with a linear combination of three sinusoids at the rotation frequency and its first two harmonics, following Queloz et al. (2009, Sect. 4), and confirm that their method is appropriate to filtering out most of the activityinduced RV variations. The residual amplitudes at the orbital periods of the planets CoRoT-7b and CoRoT-7c are used to estimate false-alarm probabilities that the RV oscillations attributed to the planets are spurious effects induced by stellar activity. For both planets we find a false-alarm probability lower than $10^{-4}$.

The presence of a third planet with a period of 9.03 days cannot be confirmed by means of our approach because this period could be associated to a harmonic of the signal with a period of 27.6 days, which can be produced by spots at high latitude on a differentially rotating star. We shall consider the effects of surface differential rotation in future models of the RV variations to investigate this issue.

Acknowledgements. The authors are grateful to an anonymous referee for a careful reading of the manuscript and several suggestions to improve their work. A.F.L. wishes to thank Drs. A.-M. Lagrange, N. Meunier, and M. Desort for interesting discussions. Active star research and exoplanetary studies at INAF-Osservatorio Astrofisico di Catania and Dipartimento di Fisica e Astronomia dell'Università degli Studi di Catania are funded by MIUR (Ministero dell'Istruzione, dell'Università e della Ricerca) and by Regione Siciliana, whose financial support is gratefully acknowledged. This research has made use of the CoRoT Public Data Archive operated at the IAS, Orsay, France, and of the ADS-CDS databases, operated at the CDS, Strasbourg, France.

\section{References}

Alonso, R., Auvergne, M., Baglin, A., et al. 2008, A\&A, 482, L21 Auvergne, M., Bodin, P., Boisnard, L., et al. 2009, A\&A, 506, 411 
A. F. Lanza et al.: Activity, rotation, and radial velocity variations of CoRoT-7

Baglin, A., Auvergne, M., Boisnard, L., et al. 2006, 36th COSPAR Scientific Assembly, 36, 3749

Barnes, J. R., Cameron, A. C., Donati, J.-F., et al. 2005, MNRAS, 357, L1

Bruntt, H., Deleuil, M., Fridlund, M., et al. 2010, A\&A, 519, A51

Chapman, G. A., Cookson, A. M., \& Dobias, J. J. 1994, ApJ, 432, 403

Croll, B., Walker, G. A. H., Kuschnig, R., et al. 2006, ApJ, 648, 607

Desort, M., Lagrange, A.-M., Galland, F., Udry, S., \& Mayor, M. 2007, A\&A, 473,983

Gray, D. F. 1988, Lectures on spectral line analysis: F, G, and K stars (Ontario: Arva)

Gray, D. F. 2009, ApJ, 697, 1032

Huber, K. F., Wolter, U., Czesla, S., et al. 2009, A\&A, 501, 715

Kurucz, R. L. 2000, http://kurucz . harvard .edu/

Lagrange, A.-M., Desort, M., \& Meunier, N. 2010, A\&A, 512, A38

Lampton, M., Margon, B., \& Bowyer, S. 1976, ApJ, 208, 177

Lanza, A. F. 2008, A\&A, 487, 1163

Lanza, A. F. 2009, A\&A, 505, 339

Lanza, A. F., Catalano, S., Cutispoto, G., Pagano, I., \& Rodonò, M. 1998, A\&A, 332,541

Lanza, A. F., Rodonò, M., Pagano, I., Barge, P., \& Llebaria, A. 2003, A\&A, 403, 1135
Lanza, A. F., Rodonò, M., \& Pagano, I. 2004, A\&A, 425, 707 Lanza, A. F., Bonomo, A. S., \& Rodonò, M. 2007, A\&A, 464, 741 Lanza, A. F., Pagano, I., Leto, G., et al. 2009a, A\&A, 493, 193

Lanza, A. F., Aigrain, S., Messina, S., et al. 2009b, A\&A, 506, 255 Léger, A., Rouan, D., Schneider, J., et al. 2009, A\&A, 506, 287 Lockwood, G. W., Skiff, B. A., Henry, G. W., et al. 2007, ApJS, 171, 260 Lomb, N. R. 1976, Ap\&SS, 39, 447

Meunier, N., Desort, M., \& Lagrange, A.-M. 2010, A\&A, 512, A39

Press, W. H., Teukolsky, S. A., Vetterling, W. T., \& Flannery, B. P. 1992, Numerical Recipes, 2nd edn. (Cambridge: Cambridge Univ. Press) Queloz, D., Henry, G. W., Sivan, J. P., et al. 2001, A\&A, 379, 279

Queloz, D., Bouchy, F., Moutou, C., et al. 2009, A\&A, 506, 303

Radick, R. R., Lockwood, G. W., Skiff, B. A., \& Baliunas, S. L. 1998, ApJS, 118,239

Saar, S. H., \& Donauhe, R. A. 1997, ApJ, 485, 319

Saar, S. H., Butler, R. P., \& Marcy, G. W. 1998, ApJ, 498, L153

Scargle, J. D. 1982, ApJ, 263, 835

Strassmeier, K. G. 2009, A\&ARv, 17, 251

Title, A. M., Topka, K. P., Tarbell, T. D., et al. 1992, ApJ, 393, 782

Vogt, S. S., \& Penrod, G. D. 1983, PASP, 95, 565 